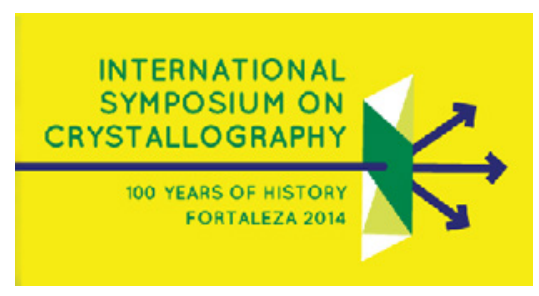

\title{
THE DEVELOPMENT OF GSAS-II
}

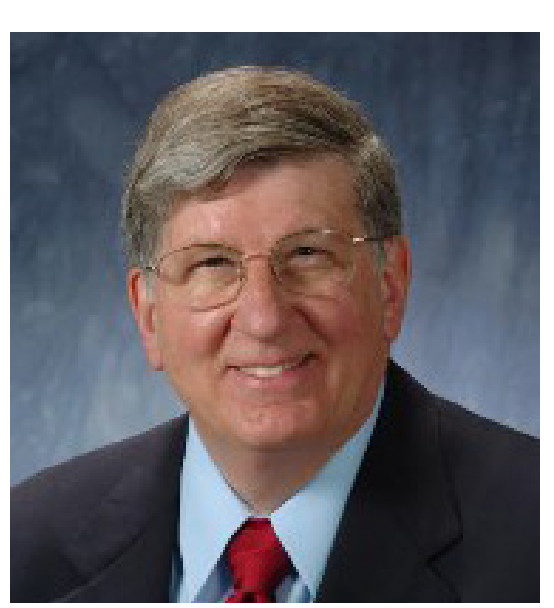

\section{Robert B. Von Dreele}

Advanced Photon Source, Argonne National Laboratory, Lemont, II, USA 60439

The General Structure Analysis System (GSAS) was originally developed more than 25 years ago to analyze multipattern single crystal and powder diffraction data sets produced at the Los Alamos spallation neutron source (LANSCE) and was written in Fortran for VAX computers. The complexity of the control file ("experiment file") required the development of an interactive editor (EXPEDT) that by means of multilayered menus allowed the user to enter values and set controls without being concerned about the details of how the records of that file were constructed. Expansion of GSAS quickly followed to include the analysis of x-ray data as well as extending the models to include e.g. complex molecular constraints and restraints, texture analysis, complex microstrain models and ultimately fitting of macromolecular structures from powder data as well as moving it to more common computer operating systems (MS Windows, linux \& Mac OSX). Meanwhile, a more modern graphical user interface (EXPGUI) was developed that allowed user interaction via a mouse as well as adding more functionality. Consequently, it now enjoys widespread use ( $>6000$ citations to date).

However, GSAS is no longer a viable platform for future development in crystal structure analysis. Thus, we have made a new start, GSAS-II, written using the Python language loaded with graphics, GUI and mathematical packages (matplotlib, pyOpenGL, wx, numpy and scipy). However, the structure and operation of Python has required new approaches to many of the algorithms used in crystal structure analysis. A very small number of old GSAS Fortran routines are imbedded within GSAS-II where computational speed was required. This talk will cover the developments for crystallographic data analysis that are now part of GSAS-II. 